\title{
Scaling the Raman gain coefficient: Applications to Germanosilicate fibers
}

\author{
Rottwitt, Karsten; Bromage, J.; Stentz, A.J.; Leng, L.; Lines, M.E.; Smith, H.
}

Published in:

Journal of Lightwave Technology

Link to article, DOI:

10.1109/JLT.2003.814386

Publication date:

2003

Document Version

Publisher's PDF, also known as Version of record

Link back to DTU Orbit

Citation (APA):

Rottwitt, K., Bromage, J., Stentz, A. J., Leng, L., Lines, M. E., \& Smith, H. (2003). Scaling the Raman gain coefficient: Applications to Germanosilicate fibers. Journal of Lightwave Technology, 21(7), 1652-1663. https://doi.org/10.1109/JLT.2003.814386

\section{General rights}

Copyright and moral rights for the publications made accessible in the public portal are retained by the authors and/or other copyright owners and it is a condition of accessing publications that users recognise and abide by the legal requirements associated with these rights.

- Users may download and print one copy of any publication from the public portal for the purpose of private study or research.

- You may not further distribute the material or use it for any profit-making activity or commercial gain

- You may freely distribute the URL identifying the publication in the public portal 


\title{
Scaling of the Raman Gain Coefficient: Applications to Germanosilicate Fibers
}

\author{
Karsten Rottwitt, Jake Bromage, Andrew J. Stentz, Lufeng Leng, Member, IEEE, Malcolm E. Lines, and Henrik Smith
}

\begin{abstract}
This paper presents a comprehensive analysis of the temperature dependence of a Raman amplifier and the scaling of the Raman gain coefficient with wavelength, modal overlap, and material composition. The temperature dependence is derived by applying a quantum theoretical description, whereas the scaling of the Raman gain coefficient is derived using a classical electromagnetic model. We also present experimental verification of our theoretical findings.
\end{abstract}

Index Terms-Optics communication, Raman amplification.

\section{INTRODUCTION}

$\mathbf{R}$ AMAN amplification is becoming increasingly important in optical communication systems as a tool for offsetting intrinsic fiber losses. During the 1980s, before the development of erbium-doped fiber amplifiers (EDFAs), much attention was given to the use of Raman amplification in optical fibers [1], [2]. There is now renewed interest for several reasons. First of all, gain is achievable at any wavelength, given a suitable pump source (1.3 $\mu \mathrm{m}$ [3], $1.4 \mu \mathrm{m}$ [4], $C$-band [5], $C$ - and $L$-band [6]). Second, broad-bandwidth amplifiers can be made by combining multiple pump wavelengths [7]. Third, and perhaps most important, the use of distributed Raman amplification in transmission spans gives better noise performance than the use of lumped amplification between the spans [8].

In this paper, we consider a fundamental parameter for Raman amplification: the Raman gain coefficient. This parameter determines the strength of the coupling between a pump beam and a signal beam due to stimulated Raman scattering. By applying the results of earlier work [9]-[13], we present a theoretical model for the Raman amplification process in optical single-mode fibers and arrive at propagation equations for signal and pump beams. Using a combination of theory and experimental data, we demonstrate how the Raman gain coefficient scales with wavelength and how the fiber temperature affects the performance of a Raman amplifier [14].

In Section II, we present a theoretical model that makes it possible to evaluate the Raman gain coefficient of an optical fiber. This model demonstrates how the fiber temperature af-

Manuscript received January 10, 2003; revised April 14, 2003.

K. Rottwitt is with the Research Center COM, Technical University of Denmark, 2800 Lyngby, Denmark.

J. Bromage and L. Leng are with OFS, Holmdel, NJ 07733 USA.

A. J. Stentz was with Bell Laboratories, Lucent Technologies, Murray Hill, NJ 07974 USA. He is now with Photuris, Piscataway, NJ 08854 USA.

M. E. Lines, retired, was with Bell Laboratoriess, Lucent Technologies, Murray Hill, NJ 07974 USA. He is now with Inplane Photonics, S. Plainfield, NJ 07080 USA.

H. Smith is with the Ørsted Laboratory, University of Copenhagen, 2100

Copenhagen, Denmark.

Digital Object Identifier 10.1109/JLT.2003.814386 fects amplifier performance. In addition, the theory provides the scaling of the gain coefficient with wavelength and with the spatial overlap of the pump and signal modes. In Section III, we compare theoretical results from Section II with experimental measurements of the wavelength and temperature dependence, and the impact on amplifier design and performance will be discussed. Section IV contains a summary of our conclusions.

\section{THEORY}

When light propagates through an optical fiber, it interacts with the fiber material. Most common fibers consist of a silica host, $\mathrm{SiO}_{2}$, to which germania, $\mathrm{GeO}_{2}$ is added in the core to increase the refractive index and form the waveguide. Such glasses are complicated since they form an amorphous network consisting of $\mathrm{Si}-\mathrm{O}-\mathrm{Si}$ and $\mathrm{Si}-\mathrm{O}-\mathrm{Ge}$ bridges and the rarer $\mathrm{Ge}-\mathrm{O}-\mathrm{Ge}$ bridges. Raman scattering results from interactions between the optical fields and this amorphous network. It is useful to imagine two atoms, silicon and/or germanium, each connected with a springlike bond to an oxygen atom. Since the oxygen atom is lighter than the other atoms, it behaves like a harmonic oscillator with an energy that is changed by the Raman process. In the most likely process, energy is transferred from the propagating field to the oscillator. As a result, the scattered light has a lower optical frequency. Afterwards, the oscillator decays toward equilibrium through a myriad of processes [12].

In order to effectively design Raman amplifiers, one must solve the propagation equation that governs an information-carrying signal beam. However, in this work, we assume that the amplifier performance may be described by a continuous signal and pump wave. This has proven experimentally to be a useful approximation. In Section II-A, we derive this equation that describes the change in photon number with distance due to the Raman process. In addition, this equation shows how temperature influences the performance of Raman amplifiers. The important material parameter of the Raman process, as described in Section II-A, is the differential polarizability. In Section II-B, we use Maxwell's equations to relate the differential polarizability to the third-order susceptibility and show how this is related to the gain of a propagating electromagnetic field. From this, we show how the Raman gain coefficient scales with wavelength and the guiding properties of an optical fiber.

\section{A. Rate Equations for the Stokes Scattering Process}

In a simple picture of the Raman scattering process where a photon at angular frequency $\omega_{p}$ scatters off a molecule, two events are possible. In one event, a phonon with the angular frequency $\omega_{v}$ is generated in addition to a photon at lower angular frequency $\omega_{s}=\omega_{p}-\omega_{v}$. This is the Stokes process. In the other 
event, the anti-Stokes process, an incoming photon receives energy from a phonon so that after the scattering process, it has a higher angular frequency $\omega_{a s}=\omega_{p}+\omega_{v}$. These events may happen spontaneously or they may be stimulated by photons at the Stokes or anti-Stokes frequencies.

In the Stokes event, the initial state, denoted by $|i\rangle$, may be described as a system with $n_{p}$ incoming photons at $\omega_{p}, n_{s}$ scattered photons at $\omega_{s}$, and $n_{v}$ phonons at $\omega_{v}$. The final state, denoted by $|f\rangle$, may be characterized as a system with $n_{p}-1$ photons at frequency $\omega_{p}, n_{s}+1$ scattered photons at $\omega_{s}$, and $n_{v}+1$ phonons at $\omega_{v}$. Alternatively, the final state may be characterized as a system with $n_{p}+1$ photons at $\omega_{p}, n_{s}-1$ scattered photons at $\omega_{s}$, and $n_{v}-1$ phonons at $\omega_{v}$. This latter process describes absorption of a photon at the Stokes shifted frequency.

In the following, $W$ denotes the steady-state rate of transitions per unit time between the initial state $|i\rangle$ and the final state $|f\rangle$. The transition rate between the initial and the final state may be evaluated from Fermi's golden rule [9]

$$
W=\frac{2 \pi}{\hbar}\left|\left\langle f\left|\hat{H}_{\text {int }}\right| i\right\rangle\right|^{2} \rho\left(\hbar \omega_{f}\right)
$$

where $\hat{H}_{\text {int }}$ is the Hamiltonian operator describing the interaction between the propagating electromagnetic fields and the scattering molecules, while $\rho\left(\hbar \omega_{f}\right)$ is the density of final states, which includes the sum of contributions from all possible final states.

We restrict our analysis to a single mode of the scattered field, as is relevant for the application of Raman scattering in an optical fiber amplifier. Starting from (1), we derive equations that govern the growth of Stokes-shifted photons within the mode. The density of states $\rho\left(\hbar \omega_{f}\right)$ depends on the damping involved in the molecular transition from the final state to the initial state. There are many processes that bring the system back to thermal equilibrium. Often a Lorentzian line shape is used for $\rho\left(\hbar \omega_{f}\right)$. For further details, see [9] and [10].

In the Raman process, the scattering molecule may be described as a quantum oscillator. The interaction Hamiltonian for a polarized dipole in an electric field equals

$$
\hat{H}_{\mathrm{int}}=-\frac{1}{2} \sum_{n} \mathbf{p}^{(R)}\left(\mathbf{r}_{n}\right) \cdot \mathbf{E}\left(\mathbf{r}_{n}\right)
$$

where $\mathbf{p}^{(R)}\left(\mathbf{r}_{n}\right)$ is the dipole moment that occurs for small molecular displacements, and $\mathbf{E}\left(\mathbf{r}_{n}\right)$ is the electric-field vector [9], [11]. In both, $\mathbf{r}_{n}$ denotes the position of the $n$th molecule. The sum extends over all molecules within the interaction volume. Note that we use SI units throughout this paper.

In this work, the dipole moment is taken to consist of two contributions, one involving the equilibrium polarizability tensor $\alpha_{i j}^{0}$ responsible for Rayleigh scattering, and the other involving the differential polarizability $\partial \alpha_{i j} / \partial q_{k}$, which is a third-rank tensor, responsible for Raman scattering [12]. The displacement of the oscillator from its equilibrium position enters the differential polarizability through the displacement vector $\mathbf{q}$, and the corresponding $i$ th component of the dipole moment is therefore given by

$$
p_{i}^{(R)}(\mathbf{r})=\sum_{j k} \frac{\partial \alpha_{i j}}{\partial q_{k}} q_{k}(\mathbf{r}) E_{j}(\mathbf{r})
$$

In the remaining part of this subsection, we describe the displacement of the oscillators by a single coordinate $q$, which constitutes a wave propagating in the same direction as the electric field (the $z$ direction)

$$
q=\sqrt{\frac{1}{N m}} \sqrt{\frac{\hbar}{2 \omega_{v}}}\left[b e^{i\left(\beta_{v} z-\omega_{v} t\right)}+b^{+} e^{-i\left(\beta_{v} z-\omega_{v} t\right)}\right] .
$$

Here, $b$ and $b^{+}$are the annihilation and creation operator of a phonon, $\omega_{v}$ is the angular frequency of a phonon, $m$ is the mass associated with the vibration, $N$ is the number of oscillators in the interaction volume $V$, and $\beta_{v}$ is the propagation constant for the phonon wave. For simplicity, we identify the number of oscillators with the number of molecules. Since we shall describe modes propagating in a waveguide, we use the propagation constant, e.g., $\beta_{v}$ in (4), and not the wave vector $k=\omega / c$. The electric field has two frequency components, one corresponding to the incoming pump field (denoted with index $p$ ), and one corresponding to the scattered field (denoted with index $s$ ). The composite quantized electric field is then

$$
\begin{aligned}
\mathbf{E}= & -i \sqrt{\frac{\hbar \omega_{p}}{2 \epsilon_{p} V}} \sum_{\sigma=1,2} \hat{\mathbf{e}}_{\sigma}^{p} \\
& \times\left[a_{p \sigma}^{+} \exp \left(-i\left(\beta_{p} z-\omega_{p} t\right)\right)\right. \\
& \left.\quad-a_{p \sigma} \exp \left(i\left(\beta_{p} z-\omega_{p} t\right)\right)\right] \\
- & i \sqrt{\frac{\hbar \omega_{s}}{2 \epsilon_{s} V}} \sum_{\sigma=1,2} \hat{\mathbf{e}}_{\sigma}^{s} \\
\times & {\left[a_{s \sigma}^{+} \exp \left(-i\left(\beta_{s} z-\omega_{s} t\right)\right)\right.} \\
& \left.\quad-a_{s \sigma} \exp \left(i\left(\beta_{s} z-\omega_{s} t\right)\right)\right]
\end{aligned}
$$

where $a_{i}$ and $a_{i}^{+}$are the annihilation and creation operators, respectively, of the photons in the scattered and the pump field $(i=s, p)$. The permittivities in (5) are approximated by the scalar quantities $\epsilon_{s}$ and $\epsilon_{p}$. The unit vectors $\hat{\mathbf{e}}_{\sigma}^{i}(i=s, p)$ specify the state of polarization (SOP) of the plane waves. The Hamiltonian (2) therefore consists of terms characterized by frequencies $\omega_{p}, \omega_{s}$, and $\omega_{v}$, and their sums and differences. We consider only the terms that are relevant for the Stokes Raman scattering process and furthermore assume that $\hat{\mathbf{e}}_{\sigma}^{p}=\hat{\mathbf{e}}_{\sigma}^{s}$ and $\hat{\mathbf{e}}_{\sigma}^{i} \cdot \hat{\mathbf{e}}_{\sigma^{\prime}}^{i}=\delta_{\sigma \sigma^{\prime}}(i=s, p)$. The interaction Hamiltonian therefore reduces to

$$
\begin{aligned}
\hat{H}_{\mathrm{int}}= & -\frac{\partial \alpha}{\partial q} \frac{\hbar^{3 / 2}}{2 V} \sqrt{\frac{1}{N m}} \sqrt{\frac{\omega_{s} \omega_{p}}{2 \omega_{v}}} \frac{1}{\sqrt{\epsilon_{s} \epsilon_{p}}} \\
\times & {\left[\sum_{\sigma=1,2}\left(a_{s \sigma}^{+} a_{p \sigma}\right) b^{+} e^{i\left\{\left(\beta_{p}-\beta_{s}-\beta_{v}\right) z-\left(\omega_{p}-\omega_{s}-\omega_{v}\right) t\right\}}\right.} \\
& +\sum_{\sigma=1,2}\left(a_{s \sigma} a_{p \sigma}^{+}\right) \\
& \left.\times b e^{-i\left\{\left(\beta_{p}-\beta_{s}-\beta_{v}\right) z-\left(\omega_{p}-\omega_{s}-\omega_{v}\right) t\right\}}\right] .
\end{aligned}
$$

For simplicity, we have represented the differential polarizability $\partial \alpha_{i j} / \partial q_{k}$ by the single component $\partial \alpha / \partial q$ (we return to the full tensor description in Section II-B). The first term represents a Raman process where a photon at $\omega_{s}$ is emitted, whereas the second term describes the absorption of a photon at $\omega_{s}$ due to the Raman process. To obey energy and momentum 
conservation laws, $\omega_{v}=\omega_{p}-\omega_{s}$ and $\beta_{v}=\beta_{p}-\beta_{s}$ for the Stokes scattering process. To simplify the notation, we replace the summations in (6) with the terms

$$
\begin{aligned}
\sum_{\sigma=1,2}\left(a_{s \sigma}^{+} a_{p \sigma}\right) b^{+} & =a_{s}^{+} a_{p} b^{+} \text {and } \\
\sum_{\sigma=1,2}\left(a_{s \sigma} a_{p \sigma}^{+}\right) b & =a_{s} a_{p}^{+} b .
\end{aligned}
$$

According to (1), the transition rate from an initial state to a final state where photons at $\omega_{s}$ are emitted is related to the matrix element

$$
\left\langle f\left|a_{s}^{+} a_{p} b^{+}\right| i\right\rangle=\sqrt{\left(n_{s}+1\right) n_{p}\left(n_{v}+1\right)}
$$

where $n_{s}$ and $n_{p}$ denote the occupation number of the scattered and incoming photons, while $n_{v}$ is the phonon occupation number. From this, we arrive at the emission rate $W_{\mathrm{em}}$ for photons at $\omega_{s}$

$$
\begin{aligned}
W_{\mathrm{em}}=\rho\left(\hbar \omega_{f}\right)\left|\frac{\partial \alpha}{\partial q}\right|^{2} & \frac{\pi \hbar^{2}}{4 V^{2} N m} \\
& \times \frac{1}{\epsilon_{s} \epsilon_{p}} \frac{\omega_{p} \omega_{s}}{\omega_{v}}\left(n_{s}+1\right) n_{p}\left(n_{v}+1\right) .
\end{aligned}
$$

Since the phonons are assumed to be in equilibrium at temperature $T$, the occupation number $n_{v}$ is the thermal equilibrium number $n_{v}=\left(\exp \left(\hbar \omega_{v} / k_{B} T\right)-1\right)^{-1}$, where $k_{B}$ is Boltzmann's constant.

Note that the emission rate scales with the product of the pump and Stokes frequencies and the phonon occupation number. The implications of this will be discussed in detail in subsequent sections.

The absorption of a photon at $\omega_{s}$ is evaluated from the second term in (6). Thus, we find

$$
\left\langle f\left|a_{s} a_{p}^{+} b\right| i\right\rangle=\sqrt{n_{s}\left(n_{p}+1\right) n_{v}} .
$$

The net rate of change per unit length of the scattered photon number at $\omega_{s}$ is the difference between the emission and absorption rates, divided by the phase velocity $v=c / \sqrt{\epsilon_{s} / \varepsilon_{0}}$, i.e.,

$$
\begin{aligned}
& \frac{d n_{s}}{d z}=C \rho\left(\hbar \omega_{f}\right) \frac{\omega_{p} \omega_{s}}{\omega_{v}} \\
& \quad \times\left\{n_{s}\left(n_{v}+1\right) n_{p}-n_{s} n_{v} n_{p}-n_{s} n_{v}+\left(n_{v}+1\right) n_{p}\right\}
\end{aligned}
$$

where the constant $C=|\partial \alpha / \partial q|^{2}\left(\pi \hbar^{2}\right) /\left(4 V^{2} N m \epsilon_{s} \epsilon_{p} v\right)$ has been introduced to simplify the notation.

Equation (11) is a rate equation for the scattered photons and is the main result of this section. It consists of four terms that are often referred to as stimulated emission, stimulated absorption, spontaneous absorption, and spontaneous emission, respectively. It should be noted that the spontaneous emission term is proportional to $n_{v}+1$ and thus depends on the temperature of the fiber. On the other hand, the difference in stimulated emission and absorption terms does not depend on the phonon number $n_{v}$ and therefore is temperature-independent. In addition, the spontaneous absorption term is typically much smaller than the other terms and can therefore be neglected.

Until now, we have focused on evaluating the number of photons at the Stokes-shifted frequency. For completeness, the rate of change in the number of anti-Stokes-shifted photons $n_{a s}$ with distance is derived by identifying terms in the Hamiltonian where $\omega_{a s}=\omega_{v}+\omega_{p}$ and $\beta_{a s}=\beta_{v}+\beta_{p}$. This leads to the result

$$
\frac{d n_{a s}}{d z} \propto \frac{\omega_{p} \omega_{a s}}{\omega_{v}}\left\{n_{p} n_{v}-\left(1+n_{v}+n_{p}\right) n_{a s}\right\} .
$$

\section{B. Gain Coefficient}

In Section II-A, we used a quantum mechanical description of the interaction between optical fields and molecular phonons to derive rate equations for the generation and amplification of Stokes photons. In this section, we make a connection to the traditional concept of nonlinear susceptibility. To make the link, we start with Maxwell's equations for classical optical fields and a classical description of molecular motion based on a forced, damped harmonic oscillator. From this, we obtain an expression for the Raman gain coefficient, including its dependence on material and field properties.

First, we consider an electric field at angular frequency $\omega_{s}$. From Maxwell's equations, we derive the relation

$$
\nabla \times \nabla \times \mathbf{E}=-\frac{1}{\varepsilon_{0} c^{2}} \frac{\partial^{2}}{\partial t^{2}} \mathbf{D} .
$$

The displacement vector $\mathbf{D}$ is related to the electric field $\mathbf{E}$ and the induced polarization $\mathbf{P}$ through the constitutive relation

$$
D_{i}=\sum_{j} \epsilon_{i j} E_{j}+P_{i}
$$

The electric permittivity tensor $\epsilon_{i j}$ is related to the first-order susceptibility tensor $\chi_{i j}^{(1)}$ through $\epsilon_{i j}=\varepsilon_{0}\left(\delta_{i j}+\chi_{i j}^{(1)}\right)$, where $\varepsilon_{0}$ is the vacuum permittivity. Note that the $i$ th component of the linear induced polarization $P_{i}^{L}=\varepsilon_{0} \sum_{j} \chi_{i j}^{(1)} E_{j}$ is included through $\chi^{(1)}$, i.e., $P_{i}$ in (14) is the nonlinear induced polarization.

As we are focusing on nonlinearity in the form of stimulated Raman scattering, we concentrate on changes in the induced polarization due to molecular motion. Here, we assume that the impact of such molecular motion is represented through the molecular polarizability, $\alpha_{i j}$. If the induced polarization oscillates at the same frequency as the applied electric field, the polarizability is a constant, represented by the equilibrium polarizability $\alpha_{i j}^{0}$. The effect of displacing atoms within a molecule may be described by including the differential polarizability $\left(\partial \alpha_{i j} / \partial q_{k}\right)$, where $q_{k}$ is the coordinate that describes the local displacement that results from the time-dependent electric field. In the case of Raman scattering, the displacement originates from an oscillating electric field and the movement of the atoms is oscillatory but may occur in different modes, such as rocking or vibrational modes [15].

The polarization induced by the electric field equals the dipole moment density and is given by

$$
\begin{aligned}
P_{i} & =\frac{N}{V} \sum_{j} \alpha_{i j} E_{j} \\
& =\frac{N}{V} \sum_{j} \alpha_{i j}^{0} E_{j}+\frac{N}{V} \sum_{j k} \frac{\partial \alpha_{i j}}{\partial q_{k}} q_{k} E_{j} .
\end{aligned}
$$

The first term, on the right-hand side (RHS), is responsible for Rayleigh scattering, whereas the second term is responsible for 
Raman scattering. Because $\mathbf{q}$ is a function of $\mathbf{E}$, the second term is nonlinear in the electric field. We show later that this term is proportional to a tensor product with the electric field to the power of three and therefore can be expressed as a third-order nonlinearity through a third-order susceptibility tensor $\chi^{(3)}$.

In the case of a Raman amplifier, the electric field may be described as a sum of two monochromatic waves, one at the pump frequency and one at the frequency of the scattered wave, which for the amplifier application, coincides with the frequency of the signal. In the following, we use indexes $s$ and $p$ to distinguish the signal and pump, respectively. If we restrict this description to the most common fiber types, i.e., weakly guiding fibers, we may separate the electric field into a transverse part $R^{i}(r)$, (where $i=s, p$ and $r=\sqrt{x^{2}+y^{2}}$ ) and a function of $z: E^{i}(z)$. The total electric field is then

$$
\begin{aligned}
\mathbf{E}=\hat{\mathbf{e}}^{p} E^{p}(z) R^{p}(r) \exp \left\{i\left(\beta_{p} z-\omega_{p} t\right)\right\} & \\
+ & +\hat{\mathbf{e}}^{s} E^{s}(z) R^{s}(r) \exp \left\{i\left(\beta_{s} z-\omega_{s} t\right)\right\}+\text { c.c. }
\end{aligned}
$$

where $\hat{\mathbf{e}}^{i}$ is a unit polarization vector and $\beta_{i}$ is the propagation constant as determined from the waveguide eigenvalue problem $\left[\nabla_{\perp}^{2}+\left(\epsilon_{i} / \varepsilon_{0}\right)\left(\omega_{i}^{2} / c^{2}\right)\right] R^{i}=\beta_{i}^{2} R^{i}$

To obtain a propagation equation for the scattered field, we assume that the displacement $\mathbf{q}$ may be expressed as

$$
\mathbf{q}=\mathbf{Q} e^{i\left(\beta_{v} z-\omega_{v} t\right)}+\text { c.c. }
$$

We then identify fields at $\omega_{s}$ appearing in the induced polarization by requiring that $\beta_{v}=\beta_{p}-\beta_{s}$ (Stokes case). From (15), the relevant term for the $i$ th component of the induced polarization at $\omega_{s}$ is then

$$
P_{i}=\frac{N}{V} e^{i\left(\beta_{s} z-\omega_{s} t\right)} \sum_{j k} \frac{\partial \alpha_{i j}}{\partial q_{k}}\left(Q_{k}\right)^{*}\left(E^{p} R^{p} \hat{e}_{j}^{p}\right)
$$

with a complex conjugate at $-\omega_{s}$.

A relation between the displacement vector, the polarizability, and the electric field may be obtained by applying a simple oscillator model to describe the Raman scattering. The oscillation of the displacement is described through the forced harmonic oscillator equation

$$
\frac{d^{2} \mathbf{q}}{d t^{2}}+2 \gamma \frac{d \mathbf{q}}{d t}+\Omega_{v}^{2} \mathbf{q}=\frac{\mathbf{F}}{m}
$$

where $\gamma$ is the damping constant and $\Omega_{v}$ is the undamped resonance frequency, $m$ the mass, and $\mathbf{F}$ the driving force of the oscillator. Since the molecular forces are conservative, the force on the oscillator is minus the gradient of the potential $W=-\overline{\mathbf{p} \cdot \mathbf{E}} / 2$, where $\mathbf{p}$ is the dipole moment given by $p_{i}=\sum_{j} \alpha_{i j} E_{j}$. The bar indicates that averaging over a few optical cycles is needed since the molecules cannot respond to optical frequencies [11]. This averaging is implicitly assumed in the remainder of this text. The force $F_{i}=-\partial W / \partial q_{i}$ is thus given by

$$
F_{k}=\frac{1}{2} \sum_{i j} \frac{\partial \alpha_{i j}}{\partial q_{k}} E_{j} E_{i} .
$$

When the total electric field interacting with the oscillator is the sum of the pump and the scattered field, the driving force at frequency $\omega_{v}$ originates from products containing the electric field at the pump and signal frequency, i.e.,
$E^{p}(z) R^{p}(r)\left(E^{s}(z) R^{s}(r)\right)^{*} \exp \left\{i\left(\beta_{v} z-\omega_{v} t\right)\right\}$. The relevant relation between the $n$th component of the force $\mathbf{F}$ and the electromagnetic field is then

$$
F_{n}=\frac{1}{2} R^{p}\left(R^{s}\right)^{*} e^{i\left(\beta_{v} z-\omega_{v} t\right)} \sum_{k l} \frac{\partial \alpha_{k l}}{\partial q_{n}} E_{k}^{p}\left(E_{l}^{s}\right)^{*}+\text { c.c. }
$$

where $E_{l}^{s}$ and $E_{k}^{p}$ are the components of the vectors $\hat{\mathbf{e}}^{s} E^{s}$ and $\hat{\mathbf{e}}^{p} E^{p}$, respectively. In (21), it is assumed that the transverse parts $R^{i}$ and $(i=s, p)$ are independent of the direction of the electric field. When the force is inserted into (19), we obtain

$$
\mathbf{Q}=\frac{\mathbf{F}_{0}}{m} \frac{1}{\left(\Omega_{v}^{2}-\omega_{v}^{2}\right)-\left(2 i \omega_{v} \gamma\right)}+\text { c.c. }
$$

where $\mathbf{F}_{0}$ is the amplitude of the force in (21). Omitting the oscillation term $\exp \left\{i\left(\beta_{s} z-\omega_{s} t\right)\right\}$, we obtain from (18) the induced polarization at frequency $\omega_{s}$ as

$$
\begin{aligned}
\left.P_{i}\right|_{\omega_{s}}=\frac{N}{2 m V} \frac{R^{s}\left|R^{p}\right|^{2}}{\left(\Omega_{v}^{2}-\omega_{v}^{2}\right)+\left(2 i \omega_{v} \gamma\right)} & \\
& \times \sum_{j n k l} \frac{\partial \alpha_{i j}}{\partial q_{n}}\left(\frac{\partial \alpha_{k l}}{\partial q_{n}} E_{k}^{p}\right)^{*} E_{l}^{s} E_{j}^{p}
\end{aligned}
$$

with a complex conjugate at frequency $-\omega_{s}$. The term that is a summation over $(k, l)$ is recognized from (21).

Alternatively, the $i$ th component of the induced third-order polarization may be written in terms of the third-order susceptibility tensor $\chi^{(3)}$ through

$$
P_{i}=\varepsilon_{0} \sum_{j k l} \chi_{i j k l}^{(3)} E_{j} E_{k} E_{l} .
$$

Assuming that the electric field can be expressed as shown in (16), the contribution oscillating at frequency $\omega_{s}$ is

$$
P_{i}=6 \varepsilon_{0}\left|R^{p}\right|^{2} R^{s} \sum_{j k l} \chi_{i j k l}^{(3)} E_{j}^{p}\left(E_{k}^{p}\right)^{*} E_{l}^{s}
$$

with a complex conjugate at $-\omega_{s}$. Comparing (23) and (25) allows us to identify

$$
\begin{aligned}
& \chi_{i j k l}^{(3)}=\frac{N}{12 m \varepsilon_{0} V} \frac{1}{\left(\Omega_{v}^{2}-\omega_{v}^{2}\right)+}\left(2 i \omega_{v} \gamma\right) \\
& \times \sum_{n} \frac{\partial \alpha_{i j}}{\partial q_{n}}\left(\frac{\partial \alpha_{k l}}{\partial q_{n}}\right)^{*} .
\end{aligned}
$$

Since an optical fiber typically is made of germanosilicate glass, which is an amorphous material, the fourth-rank tensor $\chi^{(3)}$ is isotropic. Thus, $\chi^{(3)}$ has only 21 nonzero elements, which are the elements where the indexes are either identical $\chi_{i i i i}^{(3)}$ or occur in pairs $\chi_{i j j i}^{(3)}, \chi_{i j i j}^{(3)}$, and $\chi_{i i j j}^{(3)}[12]$.

Applying (14) to (13), and assuming $\nabla \cdot \mathbf{E}=0$, (13) becomes

$$
-\nabla^{2} \mathbf{E}=\frac{n^{2}}{c^{2}} \omega_{s}^{2} \mathbf{E}+\frac{\omega_{s}^{2}}{\varepsilon_{0} c^{2}} \mathbf{P} .
$$

By inserting $\mathbf{E}=\mathbf{E}^{s}=\hat{\mathbf{e}}^{s} E^{s}(z) R^{s}(r) \exp \left\{i\left(\beta_{s} z-\omega_{s} t\right)\right\}$ from (16), and assuming that the SOP for the pump and the scattered field do not change during propagation, and neglecting $\partial^{2} \mathbf{E}^{s} / \partial z^{2}$, we obtain a wave equation for each component of the Stokes scattered wave

$$
\frac{\partial E_{i}^{s}}{\partial z} R^{s}=\frac{3 \omega_{s}^{2}}{c^{2} \beta_{s}} i R^{s}\left|R^{p}\right|^{2} \sum_{j k l} \chi_{i j k l}^{(3)} E_{j}^{p}\left(E_{k}^{p}\right)^{*} E_{l}^{s} .
$$


In an optical fiber, where the electric field is approximately transverse, i.e., $(i, j, k, l) \in(x, y)$, only four contributions appear in the sum on the RHS of (28) when the tensor properties of $\chi^{(3)}$, mentioned previously, are taken into account. Equation (28) may be further simplified by using $\chi_{i i i i}^{(3)}=\chi_{i j j i}^{(3)}+\chi_{i j i j}^{(3)}+$ $\chi_{i i j j}^{(3)}$, and $\chi_{i j i j}^{(3)}=\chi_{i i j j}^{(3)}[16]$.

Assuming that $E_{j}^{s}$ is much smaller than $E_{i}^{s}$, contributions from $\chi_{i j i j}^{(3)}$ and $\chi_{i i j j}^{(3)}$ may be neglected and (28) reduces to

$$
\begin{aligned}
& \frac{\partial E_{i}^{s}}{\partial z} R^{s}=\frac{3 \omega_{s}^{2}}{c^{2} \beta_{s}} i R^{s}\left|R^{p}\right|^{2} \\
& \times\left(\chi_{i i i i}^{(3)}\left|E_{i}^{p}\right|^{2}+\chi_{i j j i}^{(3)}\left|E_{j}^{p}\right|^{2}\right) E_{i}^{s}
\end{aligned}
$$

where $(i, j)$ is either $(x, y)$ or $(y, x)$. From this, it is clear that $\chi_{i i i i}^{(3)}$ is responsible for the Raman interaction when the pump and signal are polarized along the same axis, whereas $\chi_{i j j i}^{(3)}$ is responsible for the Raman interaction when the pump and scattered wave are polarized along orthogonal axes. Equation (29) shows that Raman gain originates from the imaginary part of the third-order susceptibility.

The use of polarization-maintaining fibers allows one to measure the two contributions in (29). In [17] and [18], such measurements show that the cross coupling is much weaker than the parallel coupling (less by more than a factor of ten) and that it peaks at a much lower frequency difference between signal and pump. This difference between cross and parallel coupling leads to polarization-dependent gain (PDG) in Raman amplifiers when the pump light has a high degree of polarization. The effect can be particularly large when the signal and pump light copropagate in the fiber [19], [20]. PDG can be significantly reduced by either depolarizing the Raman pumps with, for example, a Lyot depolarizer [21] or by using pairs of uncorrelated polarized pumps that are multiplexed with a polarization-beam combiner.

From (29), the rate equation for the power $P^{s}=$ $2 \varepsilon_{0} n_{s} c \int_{A}\left|E^{s} R^{s}\right|^{2} d A$ is

$$
\frac{d P^{s}}{d z}=4 \varepsilon_{0} n_{s} c \int_{A}\left|R^{s}\right|^{2} \operatorname{Re}\left[\left(E^{s}\right)^{*} \frac{d E^{s}}{d z}\right] d A=\gamma_{R} P^{s}
$$

where $A$ is the cross section of the fiber. This equation defines the gain coefficient $\gamma_{R}$.

If the pump is unpolarized, as is often the case, the pump power is divided equally between the two components, represented by index $i$ and $j$, at any point in time and at any point along the fiber, $2 \varepsilon_{0} n_{p} c \int_{A}\left|R^{p} E_{i}^{p}\right|^{2} d A=$ $2 \varepsilon_{0} n_{p} c \int_{A}\left|R^{p} E_{j}^{p}\right|^{2} d A=P^{p} / 2$. Consequently

$$
\begin{aligned}
\frac{d P^{s}}{d z}= & \frac{3 \omega_{s}^{2} P^{p} P^{s}}{c^{3} \beta_{s} \varepsilon_{0} n_{p}} \\
& \times \operatorname{Re}\left[\frac{\int_{A} i\left(\chi_{i i i i}^{(3)}+\chi_{i j j i}^{(3)}\right)\left|R^{p}\right|^{2}\left|R^{s}\right|^{2} d A}{\int_{A}\left|R^{p}\right|^{2} d A \int_{A}\left|R^{s}\right|^{2} d A}\right] .
\end{aligned}
$$

Equation (31) is the main result of this section. From this, one may predict the material dependence, for example, by separating the integrals into contributions from the core and from the cladding of the fiber. It also shows how to include the modal overlap of the pump and the signal.
If we furthermore assume that the susceptibilities are constant across the entire fiber cross section, the gain coefficient $\gamma_{R}$ becomes a simple average of the two contributions multiplied by the pump power

$$
\gamma_{R}=-\frac{3 \omega_{s}^{2}}{\varepsilon_{0} n_{p} c^{3} \beta_{s}} \frac{1}{A_{\mathrm{eff}}^{p s}} \frac{\operatorname{Im}\left[\chi_{i i i i}^{(3)}+\chi_{i j j i}^{(3)}\right]}{2} P^{p}
$$

where $A_{\mathrm{eff}}^{p s}$ is the effective area of the overlap between the pump and signal

$$
A_{\mathrm{eff}}^{p s}=\frac{\int_{A}\left|R^{p}\right|^{2} d A \int_{A}\left|R^{s}\right|^{2} d A}{\int_{A}\left|R^{p}\right|^{2}\left|R^{s}\right|^{2} d A} .
$$

We now make the approximation that $\beta_{s} \approx \omega_{s} \bar{n} / c$ where $\bar{n}$ is the effective refractive index of propagation. In a step-index fiber $\bar{n}$ is less than the refractive index of the core and greater than the refractive index of the cladding. From this, $\beta_{s}$ is proportional to $\omega_{s}$, and the gain coefficient $g_{R}=\gamma_{R} / P^{p}$ is given by

$$
g_{R}=-\frac{3 \omega_{s}}{\varepsilon_{0} c^{2} n_{p} n_{s}} \frac{\operatorname{Im}\left[\chi_{i i i i}^{(3)}+\chi_{i j j i}^{(3)}\right]}{2 A_{\mathrm{eff}}^{p s}} .
$$

This shows, that under the assumption that the third-order susceptibilities are frequency-independent, the gain coefficient $g_{R}$ scales linearly with the frequency of the Stokes-shifted wave. Since optical frequencies are of the order of $10^{14} \mathrm{~Hz}, \omega_{s}$ is often replaced with $\omega_{p}$ for convenience. In optical fibers, $g_{R}$ is typically of the order of $1(\mathrm{~W} \cdot \mathrm{km})^{-1}$ and $A_{\mathrm{eff}}^{p s} \approx 75 \times 10^{-12} \mathrm{~m}^{2}$.

\section{APPLICATION TO EXPERIMENTS}

From the theory previously stated, it is expected that the Raman efficiency increases as the pump wavelength decreases. However, the efficiency also depends strongly on the waveguide design and the material defining the waveguiding properties. These dependencies will be discussed in Sections III-A and B. In addition, the theory also shows that the gain in any Raman amplifier is independent of temperature, whereas the noise performance becomes worse for increasing temperature. Section III-C will focus on this temperature dependence. In Sections III-A-C, the theoretical predictions will be verified by comparing with experimental data.

\section{A. Wavelength and Fiber Dependence}

The optical properties of a light-guiding fiber, such as the refractive index and the Raman gain coefficient, depend on the constituents of the fiber. Most common fibers consist of a silica, $\mathrm{SiO}_{2}$, host to which germania, $\mathrm{GeO}_{2}$, is added in the core to increase the refractive index and form the waveguide. The distribution of germania and silica in the mixture can be calculated from the refractive index profile using Sellmeier data [22], assuming that the refractive index is a sum of independent contributions from $\mathrm{SiO}_{2}$ and $\mathrm{GeO}_{2}$. The Raman gain coefficient is determined by the number of $\mathrm{Si}-\mathrm{O}-\mathrm{Si}, \mathrm{Si}-\mathrm{O}-\mathrm{Ge}$, and $\mathrm{Ge}-\mathrm{O}-\mathrm{Ge}$ bridges and the spatial overlap of the radial profiles of pump and signal intensities, weighted by the fractional distribution of germania and silica. However, for realistic germania concentrations, the occurrence of $\mathrm{Ge}-\mathrm{O}-\mathrm{Ge}$ bridges can be neglected 

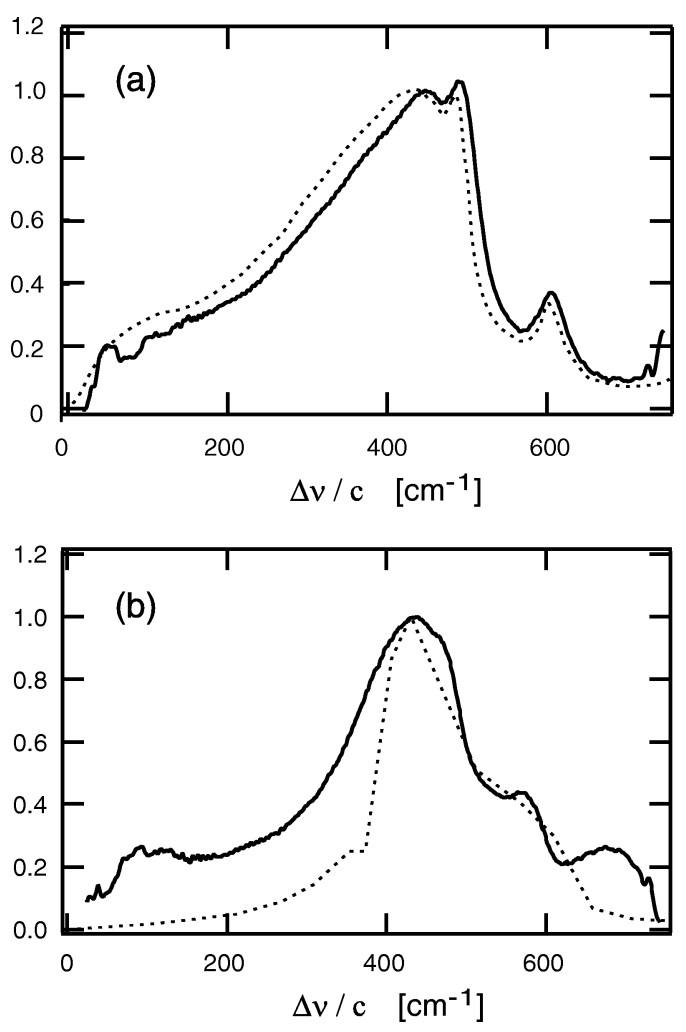

Fig. 1. Amplitude normalized spectra for (a) $C_{1-2 x}(\Delta \nu)$ and (b) $C_{2 x}(\Delta \nu)$ as a function of the separation between $1455-\mathrm{nm}$ pump and signals in $\mathrm{cm}^{-1}$. The dashed curves are measurements made on vitreous silica and germania [24].

and the relative distribution of $\mathrm{Si}-\mathrm{O}-\mathrm{Si}$ and $\mathrm{Si}-\mathrm{O}-\mathrm{Ge}$ bridges is $(1-2 x): 2 x$, where $x$ is the mole fraction $\mathrm{GeO}_{2}$ concentration. Using this, we may decompose the Raman gain spectrum into a sum of two contributions, one from $\mathrm{Si}-\mathrm{O}-\mathrm{Si}$ bridges and one from $\mathrm{Si}-\mathrm{O}-\mathrm{Ge}$ bridges.

Combining (30) and (31), we arrive at the most general expression for the gain coefficient $g_{R}$ by using $g_{R}=\gamma_{R} / P^{p}$. Assuming a fixed frequency and using the previously noted decomposition into the two material contributions, i.e., one from $\mathrm{Si}-\mathrm{O}-\mathrm{Si}$ bridges and another from $\mathrm{Si}-\mathrm{O}-\mathrm{Ge}$ bridges, we determine the Raman gain efficiency from

$$
\begin{array}{r}
g_{R}(\Delta \nu)=C_{1-2 x}(\Delta \nu) \int_{0}^{\infty}[1-2 x(r)] I^{p}(r) I^{s}(r) r d r \\
+C_{2 x}(\Delta \nu) \int_{0}^{\infty} 2 x(r) I^{p}(r) I^{s}(r) r d r
\end{array}
$$

where $I^{p}(r)$ and $I^{s}(r)$ are the radial intensity distributions for the pump and signal, respectively, normalized such that $\int_{0}^{\infty} I^{i}(r) r d r=1, i=s, p$. The quantity $\Delta \nu$ is the frequency separation between the pump and the signal. $C_{1-2 x}(\Delta \nu)$ and $C_{2 x}(\Delta \nu)$ are the material spectra for contributions to the Raman gain coefficient from $\mathrm{Si}-\mathrm{O}-\mathrm{Si}$ and $\mathrm{Si}-\mathrm{O}-\mathrm{Ge}$ bridges, respectively [23], as shown in Fig. 1. Their peak values are $4.20 \mathrm{~m}^{2} /(\mathrm{W} \cdot \mathrm{km})$ for $C_{1-2 x}$ at $445 \mathrm{~cm}^{-1}$ and $14.84 \mathrm{~m}^{2} /(\mathrm{W} \cdot \mathrm{km})$ for $C_{2 x}$ at $430 \mathrm{~cm}^{-1}$. The normalized gain coefficient spectra for vitreous silica and germania are also shown for comparison [24]. Note that the $\mathrm{Si}-\mathrm{O}-\mathrm{Si}$ and vitreous silica curves (a) are similar, as is expected. On the other hand,

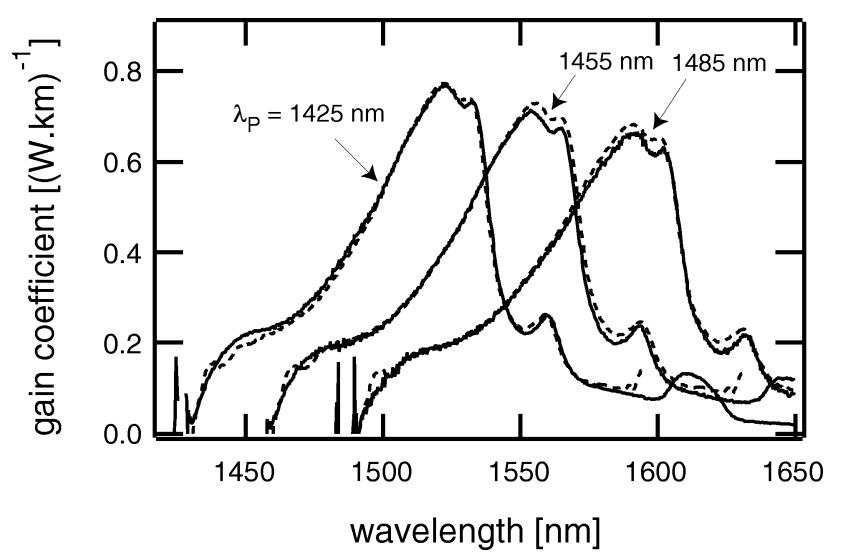

Fig. 2. Predicted (dashed curves) and measured (solid curves) Raman gain spectra on a TrueWave RS fiber spectra.

the $\mathrm{Si}-\mathrm{O}-\mathrm{Ge}$ curve (b) is significantly different from the vitreous germania curve. In particular, the feature at $675 \mathrm{~cm}^{-1}$ corresponds to a stretching vibration of the $\mathrm{Si}-\mathrm{O}-\mathrm{Ge}$ bridge and therefore is not seen in the vitreous germania spectrum [15]. This approach may be extended to include other dopants, such as fluorine or phosphor by adding additional terms to (35).

By combining the theory from Section II-B and the previously described model (35), we obtain the frequency dependence of the gain coefficient. If $\omega_{s}$ is the original frequency at which $C_{1-2 x}(\Delta \nu)$ and $C_{2 x}(\Delta \nu)$ are found, the gain coefficient at any other angular frequency $\omega_{s}^{\prime}$ becomes, according to (35)

$$
\begin{array}{r}
g_{R}(\Delta \nu)=\frac{\omega_{s}^{\prime}}{\omega_{s}} C_{1-2 x}(\Delta \nu) \int_{0}^{\infty}(1-2 x(r)) I^{p}(r) I^{s}(r) r d r \\
+\frac{\omega_{s}^{\prime}}{\omega_{s}} C_{2 x}(\Delta \nu) \int_{0}^{\infty} 2 x(r) I^{p}(r) I^{s}(r) r d r .
\end{array}
$$

For convenience, the ratio $\omega_{s}^{\prime} / \omega_{s}$ is often replaced with $\omega_{p}^{\prime} / \omega_{p}$, as mentioned in Section II-B. Using (36), the Raman gain coefficient for any germanosilicate fiber can be calculated when the refractive index profile and pump wavelength are known.

To verify this result, we measured the wavelength spectrum of the Raman gain coefficient of a transmission fiber (TrueWave RS) using three different pump wavelengths of 1425,1455 , and $1485 \mathrm{~nm}$, as shown by the solid lines in Fig. 2. (More recently, a new measurement technique has been proposed for obtaining the pump-wavelength dependence of the Raman gain coefficient [25] that does not require multiple pump wavelengths. Instead, using measurements made with a single pump, the wavelength dependence is obtained from the asymmetry between the loss from stimulated absorption at shorter wavelengths and gain from stimulated emission at longer wavelengths.) By knowing the refractive index profile of the TrueWave RS fiber and using the spectra in Fig. 1, together with (36), we were able to simulate the measurements. The results of the simulations are shown as the dashed lines in Fig. 2.

These results show that the scaling achieved in this model accurately predicts the Raman gain coefficient when one varies the pump and signal wavelengths. This example also demonstrates that the reduction in Raman efficiency with increasing pump wavelength is due in part to the reduced spatial overlap 
between the pump and signal modes. The difference between the peak Raman gain coefficients for 1425-nm and 1485-nm pumps is approximately $10 \%$, which can only be partially accounted for by the $4 \%$ reduction in $1 / \lambda_{p}$. Hence, to fully explain the reduced Raman efficiency, one must take into account the reduced overlap of pump and signal with increasing pump wavelength. Recently, results have been published for a range of modern fiber types that show that the dependence of Raman gain on the pump wavelength varies significantly depending on the fiber design [26].

\section{B. Scaling the Raman Gain Coefficient Using Effective Area}

In Section III-A, we described how one can predict the Raman gain coefficient of a single-mode germanosilicate fiber from its refractive index profile. In some cases, however, such information may not be available. In this section, we show how one may approximately scale the Raman gain coefficient from measurements made at one pump wavelength to a new pump wavelength given only information about how the effective area of the fiber changes with wavelength. This approach is helpful for designing broad-band Raman amplifiers that have many pumps covering a range of wavelengths; the Raman gain coefficient only needs to be measured at a single wavelength if one has measurements or predictions for the effective area versus wavelength.

The effective area that appears in (33) is an overlap between the spatial mode profile of the pump and signal. A more conventional effective area [14], which may typically be provided by a fiber manufacturer, is the effective area $A_{\text {eff }}(\lambda)$ of the optical mode at a single wavelength $\lambda$ as defined from the transverse part of the electric field $R(r, \lambda)$ in the following way:

$$
A_{\mathrm{eff}}(\lambda)=\frac{\left(\int_{A}|R(r, \lambda)|^{2} d A\right)^{2}}{\int_{A}|R(r, \lambda)|^{4} d A}
$$

Note that this equation is identical to (33) if $\lambda=\lambda_{p}=\lambda_{s}$. If we assume that the radial intensity distribution of the mode is Gaussian, we can write the effective area representing the overlap between pump and signal (see (33)), as [27]

$$
A_{\mathrm{eff}}^{p s}\left(\Delta \nu, \lambda_{p}\right)=\frac{A_{\mathrm{eff}}\left(\lambda_{p}\right)+A_{\mathrm{eff}}\left(\lambda_{s}\right)}{2}
$$

where the signal wavelength $\lambda_{s}$ is given by $\lambda_{s}=c /\left(c / \lambda_{p}-\right.$ $\Delta \nu)$. Using the scaling shown in (34), we can write the gain coefficient for a new pump wavelength $\Lambda_{p}$ as

$$
g_{R}\left(\Delta \nu, \Lambda_{p}\right)=g_{R}\left(\Delta \nu, \lambda_{p}\right) \cdot\left(\frac{\lambda_{s}}{\Lambda_{s}}\right) \cdot\left[\frac{A_{\mathrm{eff}}^{p s}\left(\Delta \nu, \lambda_{p}\right)}{A_{\mathrm{eff}}^{p s}\left(\Delta \nu, \Lambda_{p}\right)}\right] .
$$

The following measurement results illustrate the validity of this approach. Fig. 3 shows the measured Raman gain coefficient of a TrueWave RS fiber for four pump wavelengths: 1423.6, $1443.8,1471.3$, and $1496.0 \mathrm{~nm}$. The effective area as a function of wavelength was calculated from the refractive index profile of the fiber and is shown in Fig. 4. We used (39) to rescale each Raman gain coefficient curve from their original pump wavelengths to the new pump wavelength of $1454 \mathrm{~nm}$. The rescaled coefficients versus the frequency difference between pump and signal $\Delta \nu=\nu_{p}-\nu_{s}$ are plotted in Fig. 5. Starting with the measurements made for pump wavelengths that are $73 \mathrm{~nm}$ apart, we obtain a prediction of the Raman gain coefficient for the new

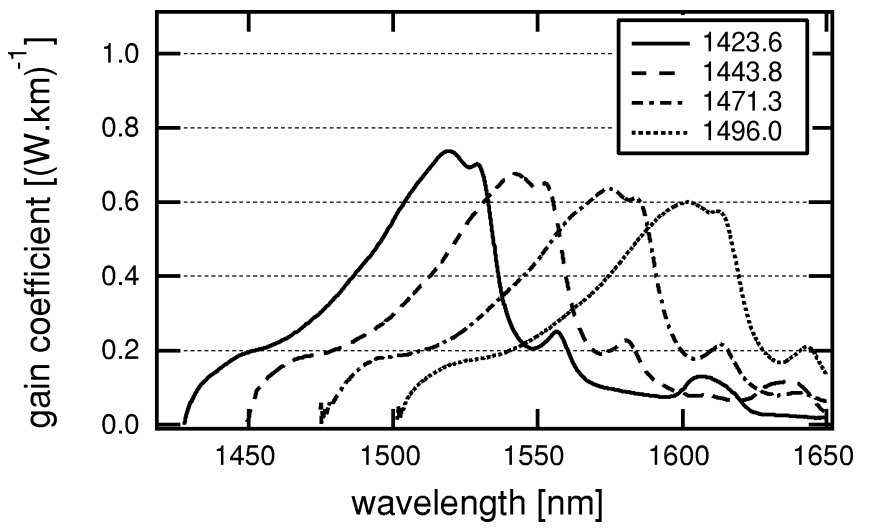

Fig. 3. Raman gain coefficients of TrueWave RS measured using four different pump wavelengths as shown in the inset.

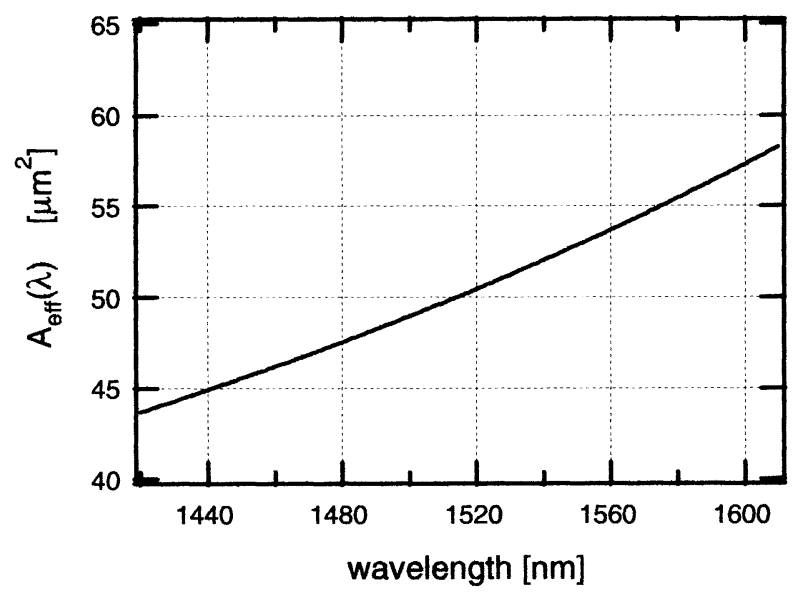

Fig. 4. Effective area as a function of wavelength. These numerical data were used in combination with (39) to test the validity of scaling Raman gain coefficients using effective area data alone.

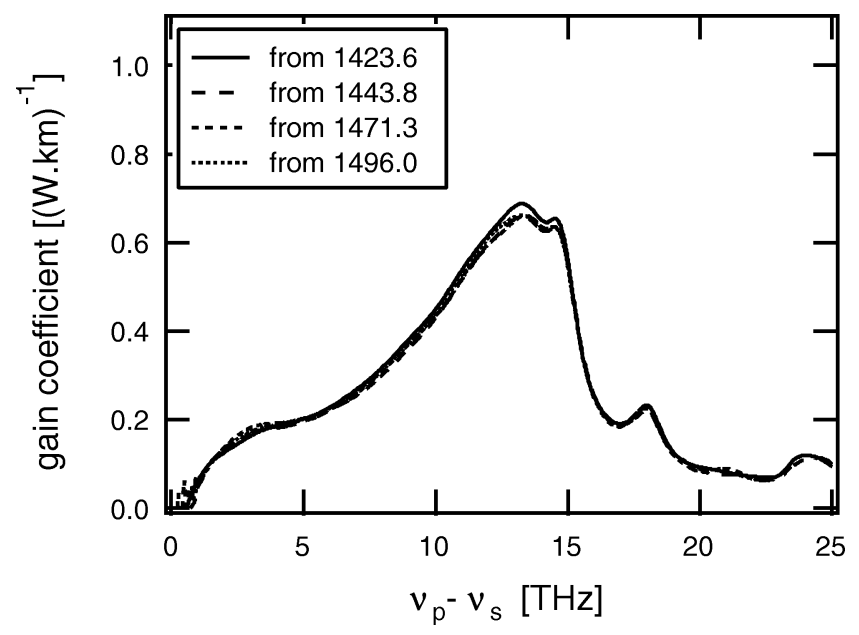

Fig. 5. Rescaled Raman gain coefficient from four different pump wavelengths to a pump at $1454 \mathrm{~nm}$.

pump wavelength that differs by only $4 \%$, confirming the validity of this approach.

\section{Temperature Dependence}

In this section, we show how the gain and noise performance of a Raman amplifier depend on the fiber temperature. As shown 
in (11), the differential equation for the increase in photons at the signal wavelength $\left(n_{s}\right)$ with $z$ has four terms. These terms correspond to emission and absorption processes that are either stimulated or spontaneous (see Section II-A). Present in each of the four terms is the phonon number $n_{v}$ at thermal equilibrium, which has the following temperature dependence for phonons at frequency $\nu$ :

$$
n_{v}=\left[\exp \left(\frac{h \nu}{k_{B} T}\right)-1\right]^{-1}
$$

as discussed following (9). Consideration of these four terms explains why Raman gain and spontaneous emission have different temperature dependencies.

Let us first consider Raman gain. As the stimulated emission and absorption terms have equal and opposite dependence on $n_{v}$, gain at the signal wavelength has no temperature dependence. Thus, if one is only interested in stimulated processes, to describe Raman gain and pump depletion in a backwardpumped amplifier with one pump and signal wavelength, one can use

$$
\begin{aligned}
& \frac{d P^{s}}{d z}=g_{R} P^{p} P^{s}-\alpha_{s} P^{s} \\
& \frac{d P^{p}}{d z}=\frac{\nu_{p}}{\nu_{s}} g_{R} P^{p} P^{s}+\alpha_{p} P^{p}
\end{aligned}
$$

where $P^{p}$ is the pump power at the frequency $\nu_{p}, P^{s}$ the signal power at frequency $\nu_{s}, \alpha_{s}$ the loss at the signal wavelength, $\alpha_{p}$ the loss at the pump wavelength, and $g_{R}$ the Raman gain coefficient.

The small-signal gain is calculated by neglecting the Raman term in the pump propagation equation that accounts for pump depletion by the signal. Often gain is expressed as an ON-OFF Raman gain $\left(G_{R}\right)$, so called because it represents the increase in signal power at the amplifier output $(z=L)$, when the pump is turned on. (Note that the small-signal ON-OFF gain is simply the net small-signal gain plus the signal span loss.) From (41) and (42), the small-signal ON-OFF gain is:

$$
G_{R}=\exp \left(g_{R} P_{L}^{p} L_{\text {eff }}\right)
$$

where $L_{\text {eff }}=\left(1-\exp \left(-\alpha_{p} L\right)\right) / \alpha_{p}$ is the effective length for the pump, and $P_{L}^{p}$ the pump power launched at $z=L$.

To model amplifier spontaneous emission noise (ASE), we include a spontaneous emission source term to (41) in accordance with (11). Denoting the ASE power in bandwidth $B_{0}$ copropagating with the signal as $P_{\mathrm{ASE}}^{+}$and in the opposite direction as $P_{\mathrm{ASE}}^{-}$, we get the equations

$$
\begin{aligned}
\pm \frac{d P_{\mathrm{ASE}}^{ \pm}}{d z}=g_{R} P^{p} & P_{\mathrm{ASE}}^{ \pm} \\
& +h \nu B_{0}\left(1+n_{v}\right) g_{R} P^{p}-\alpha_{\mathrm{ASE}} P_{\mathrm{ASE}}^{ \pm}
\end{aligned}
$$

where \pm in front of $d P_{\mathrm{ASE}}^{ \pm} / d z$ denotes power propagating in the forward and backward direction, respectively. $\alpha_{\mathrm{ASE}}$ is the fiber attenuation at the wavelength of the ASE power. The noise figure of the amplifier is

$$
F=\frac{2 \tilde{n}+1}{G}
$$

where $\tilde{n}=P_{\mathrm{ASE}} / h \nu B_{0}$ is the number of ASE photons in the signal mode, and $G$ equals the ON-OFF gain $G_{R}$ times the transmission, $T_{\mathrm{sp}}$, through the passive fiber span of the same length
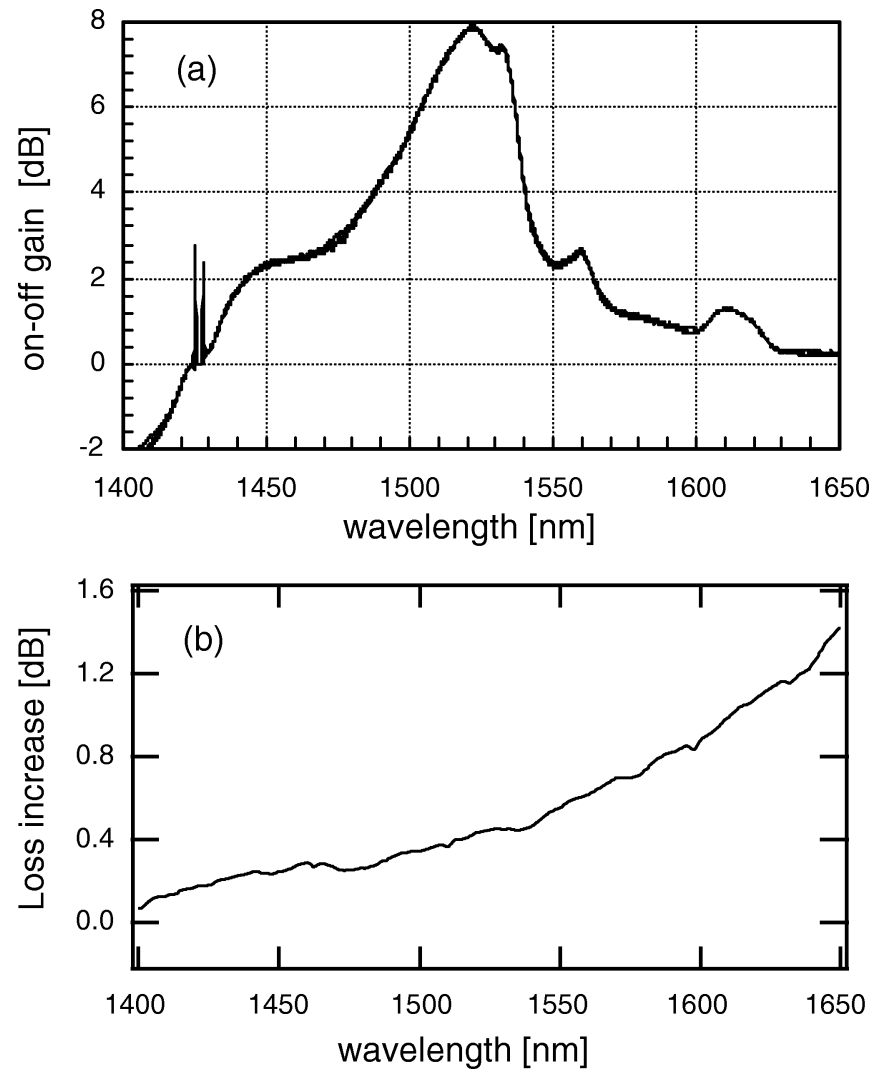

Fig. 6. (a) Small-signal ON-OFF gain at room temperature $(295 \mathrm{~K})$ and $194 \mathrm{~K}$. The two measurements are equal, illustrating that Raman gain is not temperature-dependent. (b) Increase in the background loss of the fiber due to microbends when the fiber spool was cooled.

$L$ as the distributed amplifier, i.e., $T_{\mathrm{sp}}=\exp \left(-\alpha_{s} L\right)$, as obtained from (41) by using $P^{p}=0$.

When Raman pumping is used to convert a fiber span into a distributed amplifier, the degradation of the signal-to-noise ratio (SNR) is typically much larger than that produced by a discrete amplifier. (That is, the noise figure of the pumped span may be many decibels worse than a typical discrete amplifier.) However, we can quantify the performance improvement gained by distributed amplification by calculating the noise figure that a discrete amplifier would need when placed after an unpumped span to give the same noise performance. This is called the effective noise figure $F_{\text {eff }}$ and is defined as

$$
F_{\text {eff }}=\frac{F}{T_{\mathrm{sp}}} .
$$

In the following, we report on experiments performed to test these theoretical predictions experimentally. In the first experiment, we cooled a spool containing $9 \mathrm{~km}$ of standard dispersion-shifted fiber using solid $\mathrm{CO}_{2}$ ("dry ice"). Fig. 6(a) shows the ON-OFF Raman gain measured at 295 K and 194 K. Cooling a fiber that is situated on a spool may increase the microbend loss of fiber, changing the effective length and therefore the Raman gain [see (43)]. We measured the spectral loss at both temperatures, and although we saw a significant increase in signal loss at $194 \mathrm{~K}$ [see Fig. 6(b)], the pump loss was unchanged. Furthermore, as we are interested in the small-signal $\mathrm{ON}-\mathrm{OFF}$ Raman gain, changes in signal loss are irrelevant. These 


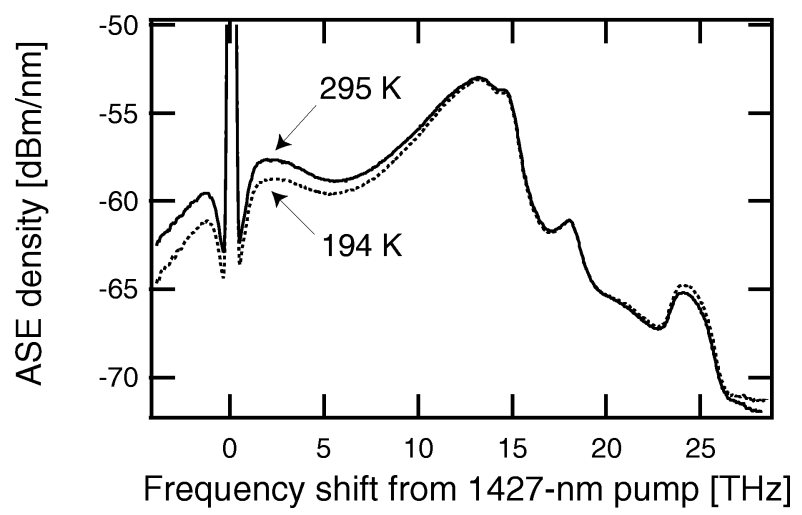

Fig. 7. Output of amplified spontaneous emission when the fiber is at $295 \mathrm{~K}$ (solid) or $194 \mathrm{~K}$ (dotted). Corresponding ON-OFF gain spectra are shown in Fig. 6. More ASE is produced when the fiber temperature is higher, particularly at wavelengths close to the pump.

measurements confirm the theoretical prediction that Raman gain does not depend on fiber temperature.

The ASE power, measured with a 1-nm resolution bandwidth is shown in Fig. 7, with the ASE power scaled to take into account increased background loss at $194 \mathrm{~K}$. These two measurements show that, unlike the Raman gain, the ASE power and, therefore, the amplifier noise figure are temperature-dependent.

To test the model quantitatively, we made measurements over a broader range of Raman gains and temperatures. Here, we counterpumped $15 \mathrm{~km}$ of dispersion-shifted fiber at $1455 \mathrm{~nm}$. We measured both the ON-OFF gain and noise figure as a function of wavelength. The first measurements were made at room temperature for peak gains ranging from 2 to $26 \mathrm{~dB}$ in $2-\mathrm{dB}$ increments. Then, we cooled the fiber to $\sim 77 \mathrm{~K}$ using liquid nitrogen and repeated the measurements.

The results are shown in Figs. 8 and 9. Here, the wavelength data are plotted as the effective noise figure versus ON-OFF gain, with different curves corresponding to different choices of peak gain. Notice that for a given value of peak gain, the noise figure is a two-valued function of gain, and so the data form a loop. This is because two wavelengths that have the same gain do not necessarily have the same effective noise figure.

If we start at signal wavelengths close to the pump and trace a loop of one curve, the noise figure increases to its maximum value. For example, for $25-\mathrm{dB}$ peak ON-OFF gain, the maximum effective noise figure is $7 \mathrm{~dB}$ for only a $3-\mathrm{dB}$ gain, at a frequency shift of about $0.4 \mathrm{THz}$ (see Fig. 8). At wavelengths further from the pump, the noise figure drops as the gain peaks at a wavelength that corresponds to a frequency close to $13 \mathrm{THz}$ below the pump. Then, for even longer wavelengths, both the gain and noise figure return to zero.

There are two effects that cause the curves to form a loop: one is the wavelength dependence of the intrinsic fiber loss and the other, and most dominant, effect is the temperature dependence of the spontaneous emission, which gives the most pronounced contribution for wavelengths close to the pump wavelength. By comparison, measurements made at $77 \mathrm{~K}$ show a much more confined loop (see Fig. 9). This is because the spontaneous emission power is reduced at lower temperatures, as theoretically predicted in Section II.

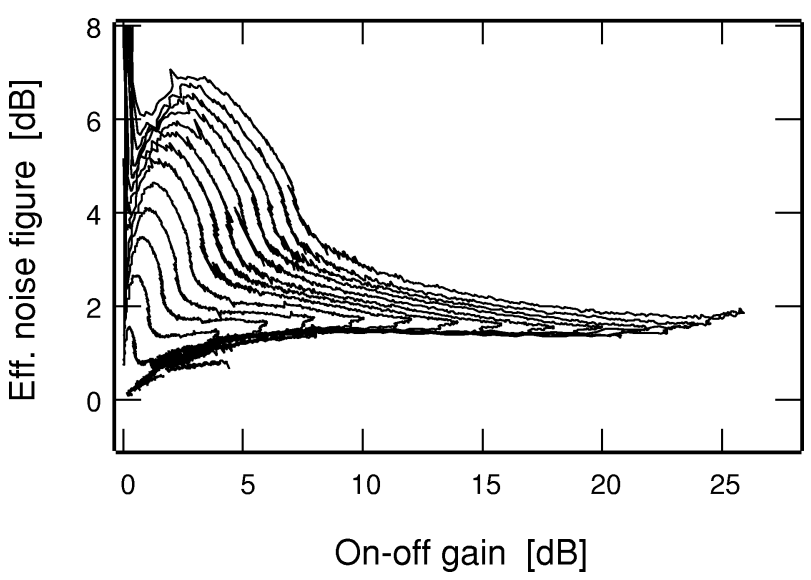

Fig. 8. Effective noise figure plotted versus ON-OFF gain for fiber at $295 \mathrm{~K}$. Each curve corresponds to a different peak gain with values ranging from 2 to $26 \mathrm{~dB}$ in $2-\mathrm{dB}$ increments.

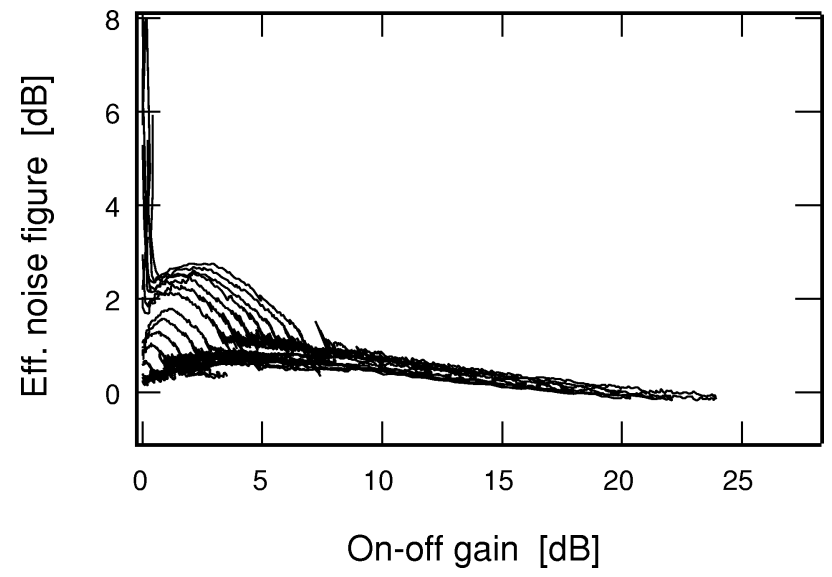

Fig. 9. Data for fiber at $77 \mathrm{~K}$. Peak gain values range from 2 to $24 \mathrm{~dB}$ in $2-\mathrm{dB}$ increments.

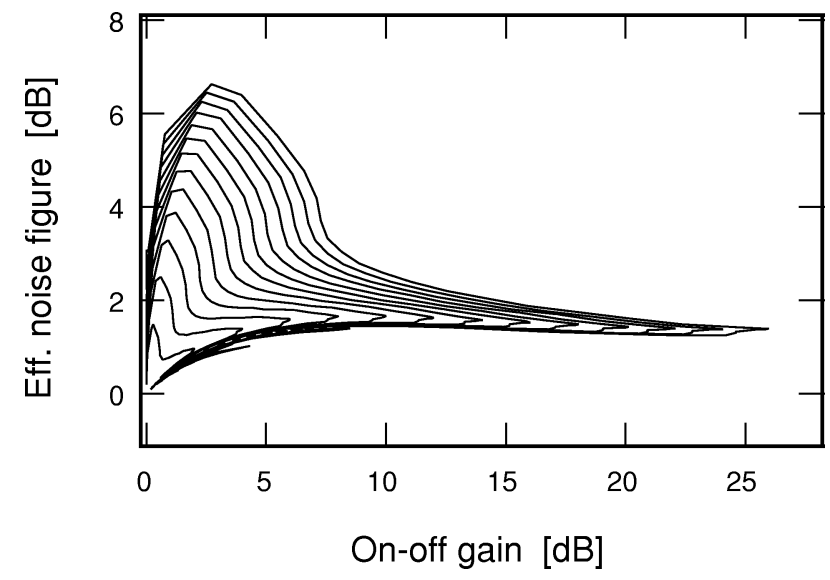

Fig. 10. Predictions for 295 K. Compare with Fig. 8.

We have used this theory in an amplifier model to accurately predict these curves for both fiber temperatures from the measured Raman gain coefficient and background losses. These predictions are shown in Figs. 10 and 11. Comparing Figs. 8-11, one can see only small deviations between measured and predicted data. The discrepancies are most significant at high values of the Raman gain. This small deviation originates from end-reflections in our measurements [28]. 


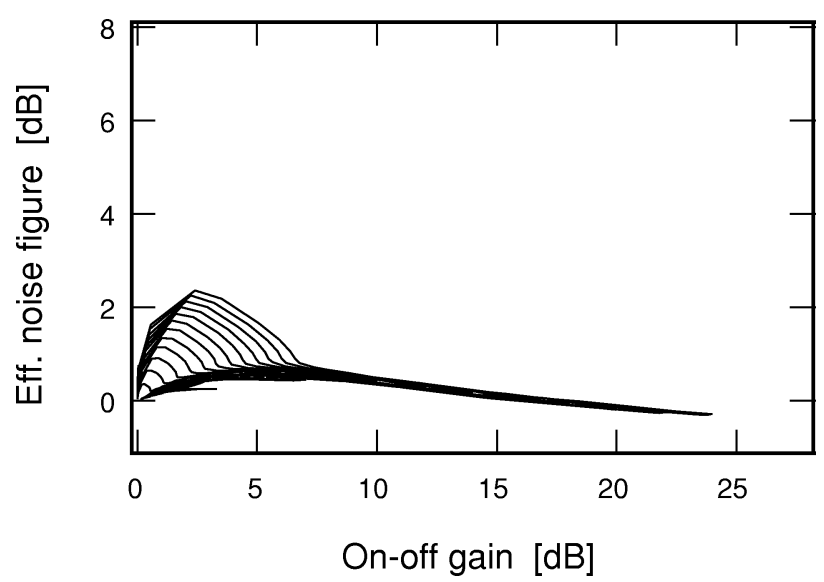

Fig. 11. Predictions for 77 K. Compare with Fig. 9.

Clearly, one must include this temperature dependence when modeling Raman amplifiers. However, for realistic temperature variations $\left(25^{\circ} \mathrm{C} \pm 50^{\circ} \mathrm{C}\right)$, the noise figure only varies by a few tenths of a decibel across commonly used signal wavelength bands. Furthermore, there is no variation in signal ON-OFF gain [28].

\section{CONCLUSION}

In this paper, we consider a critical parameter for Raman amplifier design, the Raman gain coefficient of an optical fiber. First, we present a quantum mechanical model that describes the interaction between pump and signal photons, involving optical phonons of the lattice vibrational motion. From this model, we obtain a rate equation for photons at the signal wavelength containing emission and absorption terms from both stimulated and spontaneous processes. This equation shows that Raman gain does not depend on the temperature of the fiber, whereas spontaneous emission does. We present experimental data that support these conclusions by showing excellent agreement with our numerical simulations.

In a second derivation, we model stimulated Raman scattering in terms of classical optical fields, treating the molecular system as a forced harmonic oscillator. This more intuitive model reveals the connection between the differential polarizability of a molecular lattice and its nonlinear susceptibility, a quantity traditionally used in nonlinear optics. From this result, we see why Raman gain depends on the relative polarization states of the pump and signal fields. We also explain the dependence of Raman gain on the composition of a fiber and its waveguide design. This result allows one to predict the Raman gain coefficient when the susceptibility changes radially, as is typically the case for germanosilicate optical fibers. Then, we show experimental results that support this approach. For one set of data, we compare Raman gain coefficient spectra, measured for a range of pump wavelengths, to our predictions obtained from the refractive index profile of the fiber. For another set, we showed that information about effective area versus wavelength may suffice in place of full information about the refractive index profile, if one assumes a Gaussian intensity profile for the optical mode. This approach enabled us to rescale a Raman gain measurement made for one pump wavelength to any other wavelength, using only data for the effective area of a fiber.

\section{ACKNOWLEDGMENT}

The authors would like to acknowledge valuable contributions from our colleagues at OFS, in particular from L. Grüner-Nielsen, M. O. Pedersen, B. Palsdottir, C. C. Larsen, L. E. Nelson, R. Boncek, J. J. Refi, W. A. Reed, D. Peckham, as well as J. H. Povlsen from the COM center.

\section{REFERENCES}

[1] A. Hasegawa, "Numerical study of optical soliton transmission amplified periodically by the stimulated Raman process," Appl. Opt., vol. 23, p. 3302, 1984.

[2] L. F. Mollenauer, R. H. Stolen, and M. N. Islam, "Experimental demonstration of soliton propagation in long fibers: Loss compensated by Raman gain," Opt. Lett., vol. 10, p. 229, 1985.

[3] E. M. Dianov, A. A. Abramov, M. M. Bubnov, A. V. Shipulin, A. M. Prokhorov, S. L. Semjonov, A. G. Schebunjaev, G. G. Devjatykh, A. N. Guryanov, and V. F. Khopin, "Demonstration of $1.3 \mu \mathrm{m}$ Raman fiber amplifier gain of $25 \mathrm{~dB}$ at a pumping power of $300 \mathrm{~mW}$," Opt. Fiber. Technol., vol. 1, p. 236, 1995.

[4] A. K. Srivastava, D. L. Tzeng, A. J. Stentz, J. E. Johnson, M. L. Pearsall, O. Mizuhara, T. A. Strasser, K. F. Dreyer, J. W. Sulhoff, L. Zhang, P. D. Yeates, J. R. Pedrazzani, A. M. Sergent, R. E. Tench, J. M. Freund, T. V. Nguyen, H. Manar, Y. Sun, C. Wolf, M. M. Choy, B. R. Kummer, D. Kalish, and A. R. Chraplyvy, "High speed WDM transmission in allwave fiber in both the $1.4 \mu \mathrm{m}$ and $1.55 \mu \mathrm{m}$ bands," presented at the Optical Amplifiers Their Applications, vol. PD2, Vail, CO, 1998.

[5] T. N. Nielsen, A. J. Stentz, P. B. Hansen, Z. J. Chen, D. S. Vengsarkar, T. A. Strasser, K. Rottwitt, J. H. Park, S. Stulz, S. Cabot, K. S. Feder, P. S. Westbrook, and S. G. Kosinski, " $1.6 \mathrm{tb} / \mathrm{s}(40 \times 40 \mathrm{gb} / \mathrm{s})$ transmission over $4 \times 100 \mathrm{~km}$ nonzero-dispersion fiber using hybrid Raman/erbiumdoped inline amplifiers," in Eur. Conf. Optical Communication, Nice, France, 1999, PD2-2.

[6] T. N. Nielsen, A. J. Stentz, K. Rottwitt, D. S. Vengsarkar, L. Hsu, P. B. Hansen, J. H. Park, K. S. Feder, T. A. Strasser, S. Cabot, S. Stulz, C. K. Kan, A. F. Judy, J. Sulhoff, S. Y. Park, L. E. Nelson, and L. Gruner-Nielsen, "3.28-Tb/s $(82 \times 40 \mathrm{gb} / \mathrm{s})$ transmission over $3 \times 100$ $\mathrm{km}$ nonzero-dispersion fiber using dual c- and 1-band hybrid Raman/erbium-doped inline amplifiers," presented at the Optical Fiber Communication Conf., Baltimore, MD, 2000, PD23.

[7] K. Rottwitt and H. D. Kidorf, "A 92-nm bandwidth Raman amplifier," presented at the Optical Fiber Communications Conf., San Jose, CA, 1998, PD6.

[8] P. B. Hansen, L. Eskildsen, S. G. Grubb, A. J. Stentz, T. A. Strasser, J. Judkins, J. J. DeMarco, J. J. Pedrazzani, and D. J. DiGiovanni, "Capacity upgrades of transmission systems by Raman amplification," IEEE Photon. Technol. Lett., vol. 9, pp. 262-264, Feb. 1997.

[9] A. Penzkofer, A. Laubereau, and W. Kaiser, "High intensity Raman interactions," Prog. Quantum Electron., vol. 6, p. 55, 1982.

[10] Y. R. Chen and N. Bloembergen, "Theory of stimulated Brillouin and Raman scattering," Phys. Rev., vol. 137, p. A1787, 1965.

[11] A. Yariv, Quantum Electronics. New York: Wiley, 1989.

[12] P. N. Butcher and D. Cotter, The Elements of Nonlinear Optics. Cambridge, MA: Cambridge Univ. Press, 1990.

[13] R. W. Boyd, Nonlinear Optics. San Diego, CA: Academic, 1992.

[14] G. P. Agrawal, Nonlinear Fiber Optics, 3rd ed. San Diego, CA: Academic Press, 2001, ch. 8.

[15] N. Shibata, M. Horigudhi, and T. Edahiro, "Raman spectra of binary high-silica glasses and fiber containing $\mathrm{GeO}_{2}, \mathrm{P}_{2} \mathrm{O}_{5}$ and $\mathrm{B}_{2} \mathrm{O}_{3}$," J. NonCryst. Solids, vol. 45, p. 115, 1981.

[16] M. D. Levenson and J. J. Song, "Raman-induced Kerr effect with elliptical polarization," J. Opt. Soc. Amer, vol. 66, p. 641, 1976.

[17] D. J. Dougherty, F. X. Kartner, H. A. Haus, and E. P. Ippen, "Measurement of the Raman gain spectrum of optical fibers," Opt. Lett., vol. 20, p. 31, 1995.

[18] S. Seikai, T. Nakashima, and N. Shibata, "Theory of signal light amplification by stimulated Raman scattering in twisted single-mode optical fibers," J. Lightwave Technol., vol. LT-4, pp. 583-589, June 1986. 
[19] J. Zhang, V. Dominic, M. Missey, S. Sanders, and D. Mehuys, "Dependence of Raman polarization dependent gain on pump degree of polarization at high gain levels," Optical Amplifiers and Their Applications, vol. OMB4, 2000.

[20] H. H. Kee, C. R. S. Fludger, and V. Handerek, "Statistical properties of polarization dependent gain in fiber Raman amplifiers," presented at the Optical Fiber Communications Conf., Anaheim, CA, 2002, WB2.

[21] J. S. Wang, J. R. Costelloe, and R. H. Stolen, "Reduction of the degree of polarization of a laser diode with a fiber Lyot depolarizer," IEEE Photon. Technol. Lett., vol. 11, pp. 1449-1451, Nov. 1999.

[22] M. J. Adams, An Introduction to Optical Waveguides. New York: Wiley, 1981.

[23] J. Bromage, K. Rottwitt, and M. E. Lines, "A method to predict the Raman gain spectra of germanosilicate fibers with arbitrary index profiles," IEEE Photon. Technol. Lett., vol. 14, pp. 24-26, Jan. 2002.

[24] F. L. Galeener, A. J. Leadbetter, and M. W. Stringfellow, "Comparison of the neutron, Raman, and infrared vibrational spectra of vitreous $\mathrm{SiO}_{2}$, $\mathrm{GeO}_{2}$, and $\mathrm{BeF}_{2}$," Phys. Rev. B, vol. 27, p. 1052, 1983.

[25] N. R. Newbury, "Raman gain: Pump-wavelength dependence in single-mode fiber," Opt. Lett., vol. 27, pp. 1232-1234, 2002.

[26] K. J. Cordina and C. R. S. Fludger, "Changes in Raman gain coefficient with pump wavelength in modern transmission fibers," Optical Amplifiers and Their Applications, vol. OMC3, 2002.

[27] W. P. Urquhart and P. J. Laybourn, "Effective core area of stimulated Raman scattering in single-mode optical fibers," IEE Proc., vol. 132, p. 210, 1985.

[28] K. Rottwitt, J. Bromage, M. Du, and A. J. Stentz, "Design of distributed Raman amplifiers," presented at the Eur. Conf. Optical Communication, Munich, Germany, 2000, p. 4.4.1.
Karsten Rottwitt, photograph and biography not available at the time of publication.

Jake Bromage, photograph and biography not available at the time of publication.

Andrew J. Stentz, photograph and biography not available at the time of publication.

Lufeng Leng (S'98-M'99), photograph and biography not available at the time of publication.

Malcolm E. Lines, photograph and biography not available at the time of publication.

Henrik Smith, photograph and biography not available at the time of publication. 